\title{
Role of long non-coding RNAs in normal and malignant hematopoiesis
}

\author{
WEI PanPan, HAN BoWei \& CHEN YueQin* \\ Key Laboratory of Gene Engineering of Ministry of Education, State Key Laboratory for Biocontrol, School of Life Science, Sun Yat-sen \\ University, Guangzhou 510275, China
}

Received August 2, 2013; accepted August 30, 2013; published online September 11, 2013

\begin{abstract}
Long non-coding RNAs (lncRNAs) are defined as a class of nonprotein-coding transcripts greater than 200 nucleotides in length, which have diverse functions in development and diseases including hematopoiesis. Recent advances have revealed that lncRNAs regulate hematopoietic development at almost every stage, including differentiation of the myelocyte, lymphocyte, and erythrocyte. Abnormal regulation of the lncRNAs may block aspects of blood development, which can lead to different types of hematopoietic disorders. These findings highlight the role of lncRNAs as potential therapeutic tools in malignant hematopoiesis. In this review, we summarize recent progress in the study of functional lncRNAs associated with blood development, as well as dysregulated lncRNAs involved in diverse blood diseases by interacting with crucial susceptibility genes in different pathways. In addition, we discuss genome-wide studies on lncRNAs, which are helpful for genome screening and in-depth functional study of lncRNAs associated with blood development and disease.
\end{abstract}

\section{long non-coding RNA (IncRNA), hematopoiesis, blood disease, susceptibility gene}

Citation: Wei P P, Han B W, Chen Y Q. Role of long non-coding RNAs in normal and malignant hematopoiesis. Sci China Life Sci, 2013, 56: 867-875, doi: $10.1007 / \mathrm{s} 11427-013-4550-9$

Long nonprotein-coding RNAs (lncRNAs) are generally defined as a category of nonprotein-coding transcripts more than 200 nucleotides in length. lncRNA genes cover approximately $90 \%$ of the human genome [1-4]. In recent years, it has become evident that lncRNAs are involved in a variety of cell processes [1-3,5], particularly during development and diseases [1,2,6]. Like microRNAs (miRNAs), a well-studied class of small nonprotein-coding RNAs, lncRNAs have also been shown to be highly involved in hematopoietic development, including proliferation, differentiation, and apoptosis of hematopoietic stem cells, as well as progenitors and precursors of multi-lineage mature blood cells, which include erythrocytes, megakaryocytes, myelocytes (monocyte, macrophage and neutrophil), and lymphocytes (B-cell and T-cell) [7-15]. Several findings sug- gest that dysregulation of the lncRNAs can lead to abnormal modulation of multiple cellular pathways, and result in various hematological malignancies, including lymphoma, multiple myeloma and leukemia, which together account for nearly $7 \%$ of all cancer diagnoses [12,16-19]. Dysregulation of the lncRNAs can also lead to anemia, which is the most common disorder of the blood system [14]. These involvements suggest the potential clinical relevance of IncRNAs in the diagnosis, prognosis, and therapy of these blood diseases.

lncRNAs regulate gene transcription and translation through different mechanisms, including interaction with RNA-binding proteins to reduce the translation activity of mRNA, recruiting protein complex to epigenetically regulate gene expression, and competition with mRNAs for shared miRNAs to act as post-transcriptional modifiers

*Corresponding author (email: 1sscyq@ mail.sysu.edu.cn) 
$[1-3,20]$. Although the mechanisms underlying the function of most lncRNAs are not fully understood, functional annotation has provided a complex regulation theory and classification for lncRNAs [21]. Importantly, accumulating evidence suggests that lncRNAs have multiple functions in normal and malignant hematopoiesis [11-14,16,17], and these clues help better understand the biology of hematopoiesis and blood diseases. In this review, we summarize the recent progress in our understanding of some of the mechanisms behind the involvement of lncRNAs in blood development and blood diseases through their function in modulating crucial susceptibility genes in diverse pathways. We also discuss the use of genome-wide lncRNA studies for screening potential biomarkers or therapeutic targets in blood disease treatment.

\section{IncRNAs involved in normal hematopoiesis}

Unlike mRNAs and miRNAs, lncRNAs are poorly conserved among species; however, a proportion of human lncRNAs share similar functions with murine orthologs, such as Xist, H19 and Tmevpg1 [4]. Therefore, several studies that focus on these lncRNAs in mouse model also provide important information for a better understanding of hematopoiesis. IncRNA expression levels in normal hematopoiesis have been studied in both murine and human blood cells, and a number of lineage-specific expressed lncRNAs are considered as spatiotemporal regulators of blood development [11,14] (Figure 1, left).

\subsection{Erythroid development-related IncRNAs}

Erythroid development, which is the process that produces red blood cells, is essential for the circulation system. A murine lncRNA, lincRNA-EPS, is a long intergenic non-coding RNA that was found to be highly specific to terminally differentiating erythroid cells [14]. The expression of lincRNA-EPS was strongly induced when erythroid precursors begin to synthesize hemoglobin and other lineage-specific proteins, while the inhibition of lincRNA-EPS was shown to block differentiation and promote apoptosis of erythroid progenitors, which might be related to the proapoptotic gene Pycard [14]. Briefly, lincRNA-EPS might bind to the Pycard locus and recruit transcriptionally repressed complexes to suppress the expression of Pycard. Reduced Pycard expression may not be enough to promote cell death by activating intercellular caspases, and thus normal erythroid differentiation is induced. Although the protein components associated with lincRNA-EPS in regulating erythroid differentiation are unclear, lincRNA-EPS is the first lncRNA to be related to red blood cells; however, whether this IncRNA is evolutionarily conserved in human is still unknown. Because anemia is generally caused by a lack of red blood cells or hemoglobin, lncRNAs that are involved in erythropoiesis could potentially be exploited for the treatment of anemia and other blood diseases.

\subsection{Myeloid differentiation-related IncRNAs}

Myelocytes are important in the innate immune system, and abnormal proliferation and differentiation of myeloid cells are associated with acute myeloid leukemia (AML), chronic myeloid leukemia (CML) and myelodysplastic syndrome (MDS). Eosinophil granule ontogeny (EGO) is a myeloid progenitor differentiation-related lncRNA, which is transcribed antisense to an intron of inositol triphosphate receptor type 1 (ITPRI). EGO is highly expressed in human bone marrow and in mature eosinophils [11]. Following interleukin-5 (IL-5) stimulation, EGO transcript levels increased rapidly in both $\mathrm{CD} 34^{+}$hematopoietic progenitors purified from mononuclear cord blood and bone marrow mononuclear cells, and then returned to basal level within $72 \mathrm{~h}$. It was proposed that EGO can affect myeloid development by regulating the expression of eosinophil genes, including major basic protein (MBP) and eosinophil derived neurotoxin (EDN), in developing $\mathrm{CD}_{3}{ }^{+}$hematopoietic progenitors [11]. Because EGO maintains a high expression level in the CD $34^{+}$erythroleukemic cell line TF-1, and a variety of blood diseases are caused by abnormalities of hematopoietic progenitor differentiation, especially myeloid progenitor differentiation, dysregulated or mutant EGO transcripts might serve as a potential etiology of blood diseases, such as erythroleukemia, myeloproliferative disorder, and myeloid leukemia [11].

Another lncRNA, HOTAIRM1, has been identified as an essential regulator of retinoic acid-induced myeloid cell differentiation [12]. It was found that the expression levels of HOTAIRM1 showed a positive correlation with its neighboring gene, HOXA1, and knock-down of HOTAIRM1 prevented the expression of HOXA1, HOXA4, CDI1b, and $C D 18$, which are essential transcription factors and antigens in myeloid cell differentiation. Moreover, the murine Hotairm1 was found to interact with the chromatin remodeling complexes, polycomb repressor complex 1 (PRC1) and PRC2 [22], which implies that HOTAIRM1 might maintain myeloid differentiation epigenetically in both human and murine. Because retinoic acid is an important drug in myeloid leukemia treatment [23], HOTAIRM1 might serve as a pivotal therapy target for some leukemia subtypes.

\subsection{Lymphoid differentiation and immune-related IncRNAs}

A number of lncRNAs are involved in the development and function of T-cells and B-cells, and because of the essential role of these lymphocytes in the adaptive immune system, these lncRNAs are generally involved in immune reactions.

Non-coding RNA repressor of NFAT (NRON) is present in a cytoplasmic RNA-protein scaffold complex that in- 


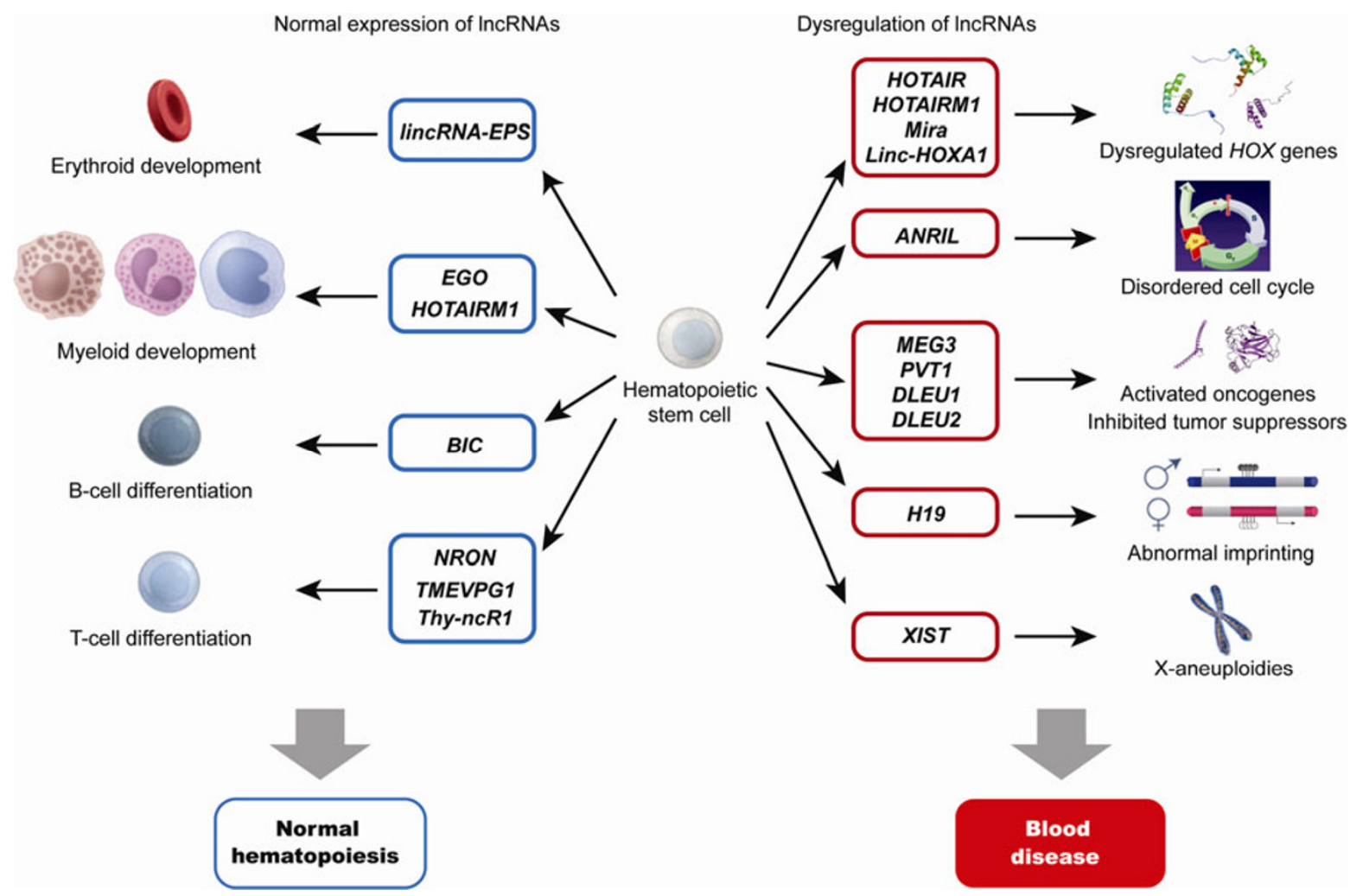

Figure 1 Normally expressed and dysregulated lncRNAs during hematopoiesis. Normally expressed lncRNAs ensure the normal differentiation of hematopoietic stem cells into erythrocytes (lincRNA-EPS), myelocytes (EGO, HOTAIRM1) and lymphocytes including B-cells (BIC) and T-cells (NRON, TMEVPG1 and Thy-ncR1), and these lncRNAs contribute to maintaining normal hematopoiesis. Dysregulated lncRNAs may disorder the biology of hematopoietic cells. For example, dysregulated HOTAIR, HOTAIRM1, Mira, or Linc-HOXA1 leads to the deregulation of HOX genes, mis-expressed ANRIL leads to disordered cell cycles, abnormal expression of MEG3, PVT1, DLEU1 or DLEU2 results in activation of oncogenes and inhibition of tumor suppressors, and dysregulated H19 and XIST leads to abnormal imprinting and X-aneuploidies, respectively. These disorders triggered by dysregulated lncRNAs result in blood diseases.

cludes phosphorylated NFAT1, a $\mathrm{Ca}^{2+}$-regulated transcription factor that controls gene expression in T-cell differentiation. Knock-down of NRON increases NFAT dephosphorylation, nuclear import (exclusively after stimulation), and production of NFAT-dependent cytokines [24]. Moreover, because lymphocytes are essential for the adaptive immune system, IncRNAs that are associated with lymphoid differentiation must also have an immune-related function. Another T-cell differentiation-related lncRNA, Thy-ncR1, is expressed specifically in cell lines derived from stage III immature T-cells, and might participate in T-cell selection and maturation by controlling the degradation of MFAP4 mRNA [13]. In addition, in the stage III immature T-cells, the $C D 1$ gene cluster, which neighbors Thy-ncR1, is also activated specifically. Although it is unclear whether the expression of Thy-ncR 1 is linked mechanistically to the activation of the $C D 1$ gene cluster, the co-expressed phenomenon of the $C D 1$ gene cluster and Thy-ncR 1 implies a potential synergistic effect of $\mathrm{CD}$ antigens and lncRNAs during T-cell selection and maturation [13]. Besides, TMEVPG1, a lncRNA studied in both murine and human, is located adjacent to the interferon-g (IFNG) gene, and induces IFN-g synthesis in a series of T-cells $[25,26]$. Con- sidering the importance of IFN-g in T-cell selection, maturation and immunization, TMEVPG1 plays multiple roles in lymphopoiesis, including driving the Th1 cell differentiation program [25] and increasing Theiler's virus persistence of activated $\mathrm{CD}^{+}$T-cells [26]. Further studies have revealed that TMEVPG1 activates IFNG by binding to WDR5, a component of the histone H3 lysine 4 (H3K4) methyltransferase complex, and altering the histone methylation level at the IFNG locus [26]. These findings suggest that lncRNAs might control T-cell differentiation and susceptibility of pathogens by regulating the abundance of pivotal cytokines at the transcriptional level.

Compared with lncRNAs in T-cells, only a few lncRNAs have been reported in B-cell differentiation. One such lncRNA is B-cell integration cluster (BIC), a lncRNA that is highly expressed in both activated B-cells and T-cells and in monocytes/macrophages; however, no evidence of BIC acting as a "long" RNA has been found yet [27]. So far, most of the studies on BIC have been focused on its processed products, miR-155-5p and miR-155-3p, which play critical roles in various physiological and pathological processes, such as hematopoietic lineage differentiation, immunity, inflammation, and viral infections [28]. Because many 
functional lncRNAs also contain miRNAs in their exons (e.g., H19 [29] and PVT-1 [30]), BIC might be involved in hematopoiesis not merely by acting as a host gene of miR-155.

In conclusion, lncRNAs play critical roles in normal hematopoiesis by regulating pivotal hematopoietic factors or cytokines, which maintain the biology balance and normal operation of the hematopoietic and immune systems, as summarized in Figure 1. Dysregulation of these lncRNAs might lead to abnormal hematopoiesis, which results in a variety of blood diseases, for example, polycythemia, thrombosis, and stroke, by reason of erythropoiesis disorder; anemia, including thalassemias and aplastic anemia; and malignant hematopoiesis, including multiple myeloma, myelodysplasia, and various subtypes of leukemia.

\section{IncRNAs associated with blood diseases in- teract with crucial susceptibility genes in diffe- rent pathways}

Many lncRNAs show cancer-specific expression [2], and the biologic features of these lncRNAs suggest that they might be involved in the pathogenesis of human diseases. Typically, lncRNAs execute molecular functions through four major known mechanisms: by acting as signals, decoys, guides, or scaffolds [31]. But in all cases, IncRNAs participate in diseases by mis-regulating target genes or pathways, which are essential factors of disease occurrence.

lncRNAs regulate these genes and pathways either in cis or in trans [1,31]. Although it is still controversial whether mammalian lncRNAs generally act in cis or in trans, both categories of lncRNAs imply a direct influence on target genes [1]. The cis-acting lncRNAs are restricted to the site of synthesis, and directly influence one or several genes on the same chromosome. They are located near diseaserelated genes, which might indicate that they have the potential to serve as disease repressors or therapy targets. By contrast, trans-acting lncRNAs diffuse across the whole genome and can influence target genes on other chromosomes, which might regulate a series of genes in a certain pathway. Studies on trans-acting IncRNAs have also provided an understanding of the possible etiology of human diseases. Emerging evidence suggests a number of lncRNAs regulate pivotal blood disease-related factors including transcriptional factors [12,32], CD antigens [12], and cyclin-dependent kinases [33,34], as well as essential hematopoietic pathways, both in cis and in trans (Figure 1, right). These findings have greatly expanded our knowledge of blood development and diseases.

\subsection{IncRNAs interact with HOX gene clusters in ma- lignant hematopoiesis}

There are a set of genes that are specifically expressed in hematopoietic stem cells, progenitors, and mature blood cells. One cluster of these genes is the highly conserved $H O X$ transcription factor genes [35]. These $H O X$ genes also have specific patterns of lineage-restricted expression, whereby HOXA genes are expressed in myeloid cells, $H O X B$ genes in erythroid cells, and HOXC genes in lymphoid cells [35]. Many lncRNAs are located in HOX loci in both human and murine, and miRNAs are found to be frequently located inside or near $\mathrm{HOX}$ loci with a 15.77 times higher incidence rate, as reported in a previous study [36]. Although there is no similar report on lncRNAs, numerous lncRNAs have been identified in $H O X$ loci, which account for a large proportion of the known lncRNAs [37]. Moreover, some of these HOX-lncRNAs have been found to play potent roles by controlling the expression of $\mathrm{HOX}$ genes at the transcriptional level [12,38-40].

One such example is HOTAIR, a lincRNA located intergenic and antisense to the flanking $\mathrm{HOXC11}$ and $\mathrm{HOXC} 12$ genes [41]. Dysregulation of HOTAIR has been shown to be involved in a variety of cancers, including breast carcinoma [42], hepatocellular carcinoma [43,44], colorectal cancer [45], and lung cancer [46]. HOTAIR binds to EZH2, a component of the H3K27 methylation complex PRC2, which leads to the genome-wide re-targeting of the PRC2 complex, and a $40-\mathrm{kb}$ region of the HOXD locus is transcriptionally silenced by HOTAIR [47]. Another example is HOTAIRM1, the myeloid differentiation-related lncRNA mentioned above, which is also found to bind with the PRC2 complex [22], and which could regulate the expression of its neighboring HOXAl gene [12]. Although it is still unclear whether the regulation of HOXAl is performed by targeting the PRC2 complex to HOXAl locus, it is certain that HOTAIRM1 plays a pivotal role in controlling HOXA gene expression. A third murine lncRNA Mira, is transcribed from a region between Hoxa6 and Hoxa7, and the Mira transcript is required for the recruitment of H3K4 histone methyltransferase Mll1 to the Mira gene locus and the activation of Hoxa6 and Hoxa7 [40]. Interestingly, not all HOX-lncRNAs regulate $H O X$ genes by regulating histone methylation. Linc-HOXA1 is a murine lncRNA adjacent to Hoxal, which represses Hoxal transcription in cis by interacting with PURB, which is an important transcriptional factor during hematopoiesis in murine embryonic stem cells [38].

It is worth noting that HOTAIR restrains the expression of HOX genes in trans and Mira activates the HOXA genes in cis $[40,47]$, which suggests that these lncRNAs located in $H O X$ clusters could control HOX gene expression by different modifications, and that linc-HOXA1 regulates HOXAl expression in a different way [38]. All these researches indicate that HOX-lncRNAs regulate $\mathrm{HOX}$ genes in a variety of different ways in complicated regulatory networks, though most parts of these networks are still unknown. Among the HOX-lncRNAs, only HOTAIRM1 has been proved to participate in hematopoiesis [12]. However, 
the HOX-lncRNAs that take part in the regulation of HOX genes and exhibit expression correlations with their neighboring HOX genes also showed a high probability of being involved in blood development [37]. There are still many HOX-lncRNAs with unknown functions. Therefore, further studies focused on HOX-lncRNAs will help in understanding the complex regulation networks of HOX-lncRNAs and $H O X$ genes, as well as functional miRNAs in the HOX locus, and might provide deeper insights into the mechanisms of normal and malignant hematopoiesis. Indeed, it is likely that these HOX-related lncRNAs represent useful therapeutic agents and targets for multiple blood diseases.

\section{2 ncRNAs control cell cycle in leukemia}

Cell cycle disorder is one of the most important causes of leukemia incidence, and numerous cyclins and cyclindependent kinases are regarded as vital oncogenes or tumor suppressors [48-50]. A cell cycle-related lncRNA, ANRIL, is located antisense of the INK4b-ARF-INK4a locus, which encode three critical cyclin-dependent kinase inhibitors, $\mathrm{p} 15^{\mathrm{INK} 4 \mathrm{~b}}, \mathrm{p} 14^{\mathrm{ARF}}$ and $\mathrm{p} 16^{\mathrm{INK} 4 \mathrm{a}}[16]$. The $\mathrm{p} 15^{\mathrm{INK} 4 \mathrm{~b}}, \mathrm{p} 14^{\mathrm{ARF}}$ and p16 ${ }^{\mathrm{INK} 4 \mathrm{a}}$ inhibitors are epigenetically silenced in hematopoietic stem cells and play central roles in cell cycle inhibition, senescence, differentiation, and stress-induced apoptosis, especially in various hematological malignancies [51], and the occurrence of $\mathrm{p} 16^{\mathrm{INK} 4 \mathrm{~A}} / \mathrm{p} 14^{\mathrm{ARF}} / \mathrm{p} 15^{\mathrm{INK} 4 \mathrm{~b}}$ alters the prognosis or disease progression in a variety of leukemia subtypes [51,52]. For example, deletion of p15 ${ }^{\text {INK4b }}$ and p16 INK4a was reported in acute lymphoblastic leukemia (ALL) (>30\%) [51] and BCR-ABL-induced leukemia [53]. Intriguingly, Iacobucci et al. [54] found a polymorphism in ANRIL locus that is associated with $B C R-A B L$-induced ALL, which might be a thread for deregulation of INK4 locus in some subtypes of leukemia. Furthermore, Yu et al. [16] discovered an inverse relation between ANRIL and p15 expression in leukemia, implying an ANRIL-dependent regulation mechanism for p15 in leukemia. This hypothesis was supported in subsequent studies. ANRIL was found to bind to SUZ12 and CBX7, which are components of PRC1 and PRC2, respectively, and recruiting these complexes to the INK4 locus, which led to the subsequent silencing of this gene locus by H3K27-trimethylation [34]. This cis-regulation of the INK4 locus by ANRIL might help explain leukemia pathogenesis, whereby, the over-expression of ANRIL epigenetically silenced p14, p15, and p16, which are repressors of leukemia, and induced cell cycle disorder, differentiation block, and apoptosis arrest in blood cells, which lead to the occurrence of leukemia. Further validation of this mechanism in hematopoietic cells might provide an effective target for the treatment of some leukemia subtypes.

ANRIL is the only cell-cycle related IncRNA that has been found to be involved in leukemia. However, other lncRNAs are known to control the cell cycle in normal so- matic cells, for example, MALAT1 in diploid fibroblasts [55], and lncRNA-LALR1 in hepatocytes [56], and in other cancer cells, for example, UCA1 in bladder carcinoma [57], and MEG3 in cervical carcinoma [58]. Further screening and research on cell-cycle related lncRNAs will also assist in targeting therapy in a series of blood diseases, especially in leukemias with cell cycle disorder.

\subsection{IncRNAs regulate oncogenes and suppressor genes in malignant hematopoiesis}

Malignant hematopoiesis is generally considered to be caused by dysregulated expression of oncogenes and tumor suppressors. Although the underlying mechanism of these abnormal expressions is unclear, lncRNAs might be an appropriate explanation of the problem. P53, which maintains genomic integrity and triggers cellular senescence, cell cycle control and apoptosis of damaged cells, is dysregulated or mutated in over half of human cancers and about $13 \%$ of hematological malignancies [59,60]. MEG3, a myelocyterelated IncRNA, is highlighted in multiple human malignancies [61] and MEG3 is a lncRNA that regulates p53, which might partially explain the aberrations of the p53 tumor suppressor pathway in some subtypes of malignant hematopoiesis. As an important regulator for proper growth and development and a putative tumor suppressor, MEG3 activates p53, inhibits cell proliferation, and might control its downstream genes by recruiting the PRC2 complex and epigenetically repressing gene expression levels [62]. MEG3 was found to display aberrant hypermethylation in its promoter region in nearly half of a cohort of $85 \mathrm{MDS}$ and AML patients [19]. Another independent assessment of 40 AML samples also confirmed the aberrant methylations [18]. The hypermethylation of the MEG3 promoter also correlates with decreased overall survival, which can serve as a prognosis marker in myeloid malignancies [19]. Because AML is a heterogeneous group of genetically and phenotypically aggressive disorders in which the differentiation of hematopoietic progenitor cells is blocked, it is highly probable that MEG3 plays an important role in myeloid differentiation. It is reasonable to assume that the interactions of MEG3 with MDM2, p53, GDF15, and p16 in other cancers will likely demonstrate a role for MEG3 that is similar to its role in leukemia [63]. These proposals need further experimental validations.

The proto-oncogene $M Y C$ is another important gene involved in malignant hematopoiesis. $M Y C$ plays a pivotal role in cell cycle regulation, metabolism, apoptosis, differentiation, cell adhesion, and tumorigenesis, and participates in regulating hematopoietic homeostasis [64]. IncRNA PVT-1 is adjacent to the MYC gene locus and is frequently involved in the translocations that occur in about $20 \%$ of Burkitt's lymphoma and $16 \%$ of multiple myeloma, as well as in most murine plasmacytomas $[30,65,66]$. Apart from 
forming fusion genes, PVT-1 is suggested to be a $M Y C$ activator, and has also been proved to be regulated by $M Y C$, which implies a feedback regulation mechanism [66]. Although details of the interaction between PVT-1 and MYC remain unrevealed, further investigation of this mechanism might provide a powerful therapy approach for malignant hematopoiesis.

In addition to the single genes, some relevant pathways are also involved in hematological malignancies. One example is the NF- $\kappa \mathrm{B}$ signaling pathway, which is centrally involved in the homeostasis of the hematopoietic system where it is induced in inflammation and inhibits apoptosis, and is activated in chronic lymphocytic leukemia (CLL) [67]. A recent study reported that two lncRNAs, DLEU1 and DLEU2, map to a critical region that is recurrently deleted in CLL [67]. In CLL patients, substantial DNAdemethylation and enriched histone $\mathrm{H} 3 \mathrm{~K} 4$ methylation at the transcriptional start sites of DLEU1 and DLEU2 were found. These two lncRNAs might control a series of genes in the NF- $\kappa \mathrm{B}$ pathway, either by inducing or repressing the pathway activity, implying that DLEU1 and DLEU2 exert a precise and complicated NF- $\kappa \mathrm{B}$ pathway regulating mechanism [67]. In brief, some lncRNAs regulate pivotal oncogenes, tumor suppressors or relevant pathways in malignant hematopoiesis, which suggests an important focus of further study, even though, at present, little is known about these mechanisms.

\subsection{IncRNAs interact with imprinted genes in hemato- logical malignancies}

Abnormal imprinting is also an important pathogenesis in many blood diseases $[68,69]$. One of the earliest described lncRNAs, H19, is involved in imprinting regulation of its neighboring gene $I G F 2$, as well as in trans-regulation of a group of imprinted genes, including Igf2 $r$ and DlkI [70,71], suggesting an important role for $\mathrm{H} 19$ in genomic imprinting during growth and development. H19 is considered to be an oncogene or tumor suppressor in different cancers [71-74]. In malignant hematopoiesis, expression of H19 is reduced in AML and CML [75,76], as well as in chronic MDS [75]. Loss of imprinting at the H19 locus that results in high H19 expression was found in adult T-cell leukemia and lymphoma patients [77], suggesting that H19 plays disparate roles in different leukemia subtypes. Accumulating evidence suggests that H19 could bind PRC2 [62] and Igf2 mRNA binding-protein 1 (IMP1) [78], which could regulate gene expression levels by both chromatin remodeling and post-transcriptional processes. Moreover, H19 also contains the precursor of miR-675 in exon one [29], suggesting that H19 might serve as a bi-functional RNA which can act either as a miRNA or an lncRNA in different regulation pathways and cell contexts. This makes the regulation mechanism of H19 more complicated, and might help to explain the conflicting reports that it acts as both a tumor suppressor and oncogene in malignant hematopoiesis. Because of its widespread and complicated roles in blood disease, further research to identify how H19 functions as a full-length lncRNA or miR-675 in different cell contexts might reveal the underlying mechanisms of H19 regulation. Further identification of target genes of both lncRNA H19 and miR-675 in malignant hematopoiesis could also provide potential specific therapeutic targets for different subtypes of leukemia. In addition, H19 is regarded as a prognosis marker in multiple cancers $[73,74,79,80]$, and the prognostic implication of H19 in blood disease is also worth exploring to develop lncRNA-based diagnosis and lncRNAmediated therapy.

\subsection{IncRNAs associated with X-chromosome inactiva- tion in hematological malignancies}

A large number of lncRNAs are located in the X chromosome and participate in $\mathrm{X}$ chromosome inactivation, including XIST, TSIX, JPX, and XITE [1]. These X chromosome-inactive related lncRNAs silence gene expression by the epigenetic modification of histones and DNA in a complicated interaction model. X chromosome aneuploidies, on the other hand, have long been associated with human cancers, but the causality was not established until recently [17]. Yildirim et al. [17] deleted Xist in the blood of mice and demonstrated that mutant females develop a highly aggressive myeloproliferative neoplasm and MDS with 100\% penetrance, which suggested that Xist loss results in X reactivation and induces significantly higher abundance of $\mathrm{X}$-linked genes, including some oncogenes. Thus, it was confirmed that Xist is required not only to maintain $\mathrm{X}$ chromosome inactivation, but is also necessary in suppressing cancer in vivo. Although no studies on other X chromosome-inactive-related lncRNAs in blood diseases have been performed, because abnormal $\mathrm{X}$ chromosome inactivation might cause various blood diseases, it is possible that these $\mathrm{X}$ chromosome-inactive-related lncRNAs might also be involved in blood disease suppressing.

To summarize, a great many nonprotein-coding transcripts that are relevant in normal and malignant hematopoiesis have been found to regulate their target genes by RNA interference or silencing at the chromatin level, as depicted in Figure 1. However, our knowledge of these lncRNAs is just the tip of the iceberg; thus, further research and information mining about these lncRNAs are still required.

\section{Genome identification of IncRNAs might provide new targets for blood diseases}

High-throughput sequencing and microarray analysis have provided powerful tools to obtain global cell transcriptome 
profiles, which can be used to screen potential lncRNAs with physiological functions in the biology of blood diseases $[81,82]$. These genomic studies will also provide databases for further functional studies. A transcriptome sequencing study of the Sezary syndrome, one of the most common forms of cutaneous T-cell lymphoma, was performed recently by Lee et al. [83]. Thirteen unannotated lncRNAs were found to be differentially expressed in Sezary syndrome. The functional annotation of these Sezary syndrome-associated lncRNAs will provide further insights into the mechanisms of Sezary syndrome pathogeny. Although only a small number of genome-wide studies of this type have been performed till now, such studies may detect potent IncRNAs involved in leukemia classification and therapy.

Another class of genome-wide studies on lncRNAs is the screening of DNA and histone modifications, and transcription factor binding sites by chromatin immunoprecipitation followed by sequencing (ChIP-seq), which has provided the modification profiles of lncRNAs [81,84]. These data have contributed to in-depth knowledge of the spatiotemporal expression pattern of IncRNAs, which has provided further clues and evidence for understanding the function of blood disease-specific lncRNAs. Although some ChIP-seq data for blood cell lines have been provided by the ENCODE project [85], ChIP-seq of patients with blood diseases and of cells under different treatments are still important for lncRNA screening. In addition, the re-analysis of previous ChIP-seq data that were focused on mRNAs may also help in identifying lncRNAs associated with various blood diseases.

A third class of genome-wide studies is RNA immunoprecipitation followed by sequencing (RIP-seq) [62]. Many lncRNAs bind to protein complexes, therefore, the RIP-seq studies on different cells could indicate potential functions of lncRNAs that bind with known complexes. A combined analysis of RNA expression data and ChIP-seq data might help define the function of some lncRNAs. Genome-wide data provides the opportunity for researchers to select pivotal IncRNAs in blood disease, and to predict the potent functional mechanism of these IncRNAs. Thus far, only a few genome-wide lncRNA studies on blood disease have been performed and future genome-wide research on lncRNAs would help to explain the mechanisms of lncRNAs, which may contribute to the diagnosis and treatment of blood diseases.

\section{Conclusion and perspectives}

Emerging studies have defined lncRNAs as an important aspect of development, and accumulating evidence suggests that IncRNAs play central roles in hematopoiesis; however, the mechanisms of action of the majority of lncRNAs in normal and malignant hematopoiesis are still unclear. Fu- ture genome-wide screening and in-depth functional studies on the IncRNAs are urgently needed to provide insights into the interaction networks of lncRNAs, mRNAs, and miRNAs in the biology of normal and malignant hematopoiesis. Such studies might offer powerful clinical tools and novel molecular strategies for blood disease diagnosis, prognosis and therapy.

This work was supported by the National Basic Research Program of China (2011CB8113015, 2011CBA0110) and National Natural Science Foundation of China (81270629).

1 Lee J T. Epigenetic regulation by long noncoding RNAs. Science, 2012, 338: 1435-1439

2 Wapinski O, Chang H Y. Long noncoding RNAs and human disease. Trends Cell Biol, 2011, 21: 354-361

3 Hung T, Chang H Y. Long noncoding RNA in genome regulation: Prospects and mechanisms. RNA Biol, 2010, 7: 582-585

4 Guttman M, Amit I, Garber M, et al. Chromatin signature reveals over a thousand highly conserved large non-coding RNAs in mammals. Nature, 2009, 458: 223-227

5 Tsai M C, Spitale R C, Chang H Y. Long intergenic noncoding RNAs: New links in cancer progression. Cancer Res, 2011, 71: 3-7

6 Spizzo R, Almeida M I, Colombatti A, et al. Long non-coding RNAs and cancer: A new frontier of translational research? Oncogene, 2012, 31: 4577-4587

7 Vasilatou D, Papageorgiou S, Pappa V, et al. The role of microRNAs in normal and malignant hematopoiesis. Eur J Haematol, 2010, 84: $1-16$

8 Sun Y M, Lin K Y, Chen Y Q. Diverse functions of miR-125 family in different cell contexts. J Hematol Oncol, 2013, 6: 6

9 O'Connell R M, Baltimore D. microRNAs and hematopoietic cell development. Curr Top Dev Biol, 2012, 99: 145-174

10 Garzon R, Pichiorri F, Palumbo T, et al. microRNA fingerprints during human megakaryocytopoiesis. Proc Natl Acad Sci USA, 2006, 103: 5078-5083

11 Wagner L A, Christensen C J, Dunn D M, et al. EGO, a novel, noncoding RNA gene, regulates eosinophil granule protein transcript expression. Blood, 2007, 109: 5191-5198

12 Zhang X, Lian Z, Padden C, et al. A myelopoiesis-associated regulatory intergenic noncoding RNA transcript within the human HOXA cluster. Blood, 2009, 113: 2526-2534

13 Aoki K, Harashima A, Sano M, et al. A thymus-specific noncoding RNA, Thy-ncR1, is a cytoplasmic riboregulator of MFAP4 mRNA in immature T-cell lines. BMC Mol Biol, 2010, 11: 99

$14 \mathrm{Hu} \mathrm{W}$, Yuan B, Flygare J, et al. Long noncoding RNA-mediated anti-apoptotic activity in murine erythroid terminal differentiation. Genes Dev, 2011, 25: 2573-2578

15 Han B W, Chen Y Q. Potential pathological and functional links between long noncoding RNAs and hematopoiesis. Science signaling, 2013, 6: re5

$16 \mathrm{Yu} \mathrm{W}$, Gius D, Onyango P, et al. Epigenetic silencing of tumour suppressor gene p15 by its antisense RNA. Nature, 2008, 451: 202-206

17 Yildirim E, Kirby J E, Brown D E, et al. Xist RNA is a potent suppressor of hematologic cancer in mice. Cell, 2013, 152: 727-742

18 Khoury H, Suarez-Saiz F, Wu S, et al. An upstream insulator regulates DLK1 imprinting in AML. Blood, 2010, 115: 2260-2263

19 Benetatos L, Hatzimichael E, Dasoula A, et al. CpG methylation analysis of the MEG3 and SNRPN imprinted genes in acute myeloid leukemia and myelodysplastic syndromes. Leuk Res, 2010, 34: 148-153

20 Poliseno L, Salmena L, Zhang J, et al. A coding-independent function of gene and pseudogene mRNAs regulates tumour biology. Nature, 2010, 465: 1033-1038 
21 Qi P, Du X. The long non-coding RNAs, a new cancer diagnostic and therapeutic gold mine. Mod Pathol, 2012, 26: 155-165

22 Guttman M, Donaghey J, Carey B W, et al. lincRNAs act in the circuitry controlling pluripotency and differentiation. Nature, 2011, 477: 295-300

23 Ortega J J, Madero L, Martin G, et al. Treatment with all-trans retinoic acid and anthracycline monochemotherapy for children with acute promyelocytic leukemia: A multicenter study by the pethema group. J Clin Oncol, 2005, 23: 7632-7640

24 Sharma S, Findlay G M, Bandukwala H S, et al. Dephosphorylation of the nuclear factor of activated $t$ cells (NFAT) transcription factor is regulated by an RNA-protein scaffold complex. Proc Natl Acad Sci USA, 2011, 108: 11381-11386

25 Collier S P, Collins P L, Williams C L, et al. Cutting edge: Influence of Tmevpg1, a long intergenic noncoding RNA, on the expression of Ifng by Th1 cells. J Immunol, 2012, 189: 2084-2088

26 Gomez J A, Wapinski O L, Yang Y W, et al. The NEST long ncRNA controls microbial susceptibility and epigenetic activation of the interferon-gamma locus. Cell, 2013, 152: 743-754

27 Eis P S, Tam W, Sun L, et al. Accumulation of miR-155 and BIC RNA in human B cell lymphomas. Proc Natl Acad Sci USA, 2005, 102: $3627-3632$

28 Elton T S, Selemon H, Elton S M, et al. Regulation of the miR155 host gene in physiological and pathological processes. Gene, 2012, doi: 10.1016/j.gene.2012.12.009

29 Cai X, Cullen B R. The imprinted H19 noncoding RNA is a primary microRNA precursor. RNA, 2007, 13: 313-316

30 Beck-Engeser G B, Lum A M, Huppi K, et al. Pvt1-encoded microRNAs in oncogenesis. Retrovirology, 2008, 5: 4

31 Wang K C, Chang H Y. Molecular mechanisms of long noncoding RNAs. Mol Cell, 2011, 43: 904-914

32 Gupta R A, Shah N, Wang K C, et al. Long non-coding RNA HOTAIR reprograms chromatin state to promote cancer metastasis. Nature, 2010, 464: 1071-1076

33 Kotake Y, Nakagawa T, Kitagawa K, et al. Long non-coding RNA ANRIL is required for the PRC2 recruitment to and silencing of p15 (INK4B) tumor suppressor gene. Oncogene, 2011, 30: 1956-1962

34 Yap K L, Li S, Munoz-Cabello A M, et al. Molecular interplay of the noncoding RNA ANRIL and methylated histone $\mathrm{H} 3$ lysine 27 by polycomb CBX7 in transcriptional silencing of INK4A. Mol Cell, 2010, 38: 662-674

35 Alharbi R A, Pettengell R, Pandha H S, et al. The role of HOX genes in normal hematopoiesis and acute leukemia. Leukemia, 2012, 27: 1000-1008

36 Calin G A, Sevignani C, Dumitru C D, et al. Human microRNA genes are frequently located at fragile sites and genomic regions involved in cancers. Proc Natl Acad Sci USA, 2004, 101: 2999-3004

37 Sasaki Y T, Sano M, Kin T, et al. Coordinated expression of ncRNAs and HOX mRNAs in the human HOXA locus. Biochem Biophys Res Commun, 2007, 357: 724-730

38 Maamar H, Cabili M N, Rinn J, et al. Linc-HOXA1 is a noncoding RNA that represses Hoxa1 transcription in cis. Genes Dev, 2013, 27: 1260-1271

39 Wang K C, Yang Y W, Liu B, et al. A long noncoding RNA maintains active chromatin to coordinate homeotic gene expression. Nature, 2011, 472: 120-124

40 Bertani S, Sauer S, Bolotin E, et al. The noncoding RNA Mistral activates hoxa6 and hoxa7 expression and stem cell differentiation by recruiting MLL1 to chromatin. Mol Cell, 2011, 43: 1040-1046

41 Rinn J L, Kertesz M, Wang J K, et al. Functional demarcation of active and silent chromatin domains in human HOX loci by noncoding RNAs. Cell, 2007, 129: 1311-1323

42 Lu L, Zhu G, Zhang C, et al. Association of large noncoding RNA HOTAIR expression and its downstream intergenic $\mathrm{CpG}$ island methylation with survival in breast cancer. Breast Cancer Res Treat, 2012, 136: 875-883

43 Ishibashi M, Kogo R, Shibata K, et al. Clinical significance of the expression of long non-coding RNA HOTAIR in primary hepatocellular carcinoma. Oncol Rep, 2013, 29: 946-950
44 Geng Y J, Xie S L, Li Q, et al. Large intervening non-coding RNA HOTAIR is associated with hepatocellular carcinoma progression. J Int Med Res, 2011, 39: 2119-2128

45 Kogo R, Shimamura T, Mimori K, et al. Long noncoding RNA HOTAIR regulates polycomb-dependent chromatin modification and is associated with poor prognosis in colorectal cancers. Cancer Res, 2011, 71: 6320-6326

46 Zhuang Y, Wang X, Nguyen H T, et al. Induction of long intergenic non-coding RNA HOTAIR in lung cancer cells by type I collagen. $\mathrm{J}$ Hematol Oncol, 2013, 6: 35

47 Tsai M C, Manor O, Wan Y, et al. Long noncoding RNA as modular scaffold of histone modification complexes. Science, 2010, 329: 689-693

48 Bonn B R, Krieger D, Burkhardt B. Cell cycle regulatory molecular profiles of pediatric T-cell lymphoblastic leukemia and lymphoma. Leuk Lymphoma, 2012, 53: 557-568

49 Geyer C R. Strategies to re-express epigenetically silenced p15 (INK4B) and p21 (WAF1) genes in acute myeloid leukemia. Epigenetics, 2010, 5: 696-703

50 Renner K, Ausserlechner M J, Kofler R. A conceptual view on glucocorticoid-induced apoptosis, cell cycle arrest and glucocorticoid resistance in lymphoblastic leukemia. Curr Mol Med, 2003, 3: 707-717

51 Drexler H G. Review of alterations of the cyclin-dependent kinase inhibitor INK4 family genes p15, p16, p18 and p19 in human leukemia-lymphoma cells. Leukemia, 1998, 12: 845-859

52 Tsihlias J, Kapusta L, Slingerland J. The prognostic significance of altered cyclin-dependent kinase inhibitors in human cancer. Annu Rev Med, 1999, 50: 401-423

53 Williams R T, Sherr C J. The INK4-ARF (CDKN2A/B) locus in hematopoiesis and BCR-ABL-induced leukemias. Cold Spring Harb Symp Quant Biol, 2008, 73: 461-467

54 Iacobucci I, Sazzini M, Garagnani P, et al. A polymorphism in the chromosome 9p21 ANRIL locus is associated to Philadelphia positive acute lymphoblastic leukemia. Leuk Res, 2011, 35: 1052-1059

55 Tripathi V, Shen Z, Chakraborty A, et al. Long noncoding RNA MALAT1 controls cell cycle progression by regulating the expression of oncogenic transcription factor B-MYB. PLoS Genet, 2013, 9: e1003368

56 Xu D, Yang F, Yuan J H, et al. Long noncoding RNAs associated with liver regeneration 1 accelerates hepatocyte proliferation during liver regeneration by activating Wnt/beta-Catenin signaling. Hepatology, 2013, 58: 739-751

57 Yang $\mathrm{C}$, Li X, Wang Y, et al. Long non-coding RNA UCA1 regulated cell cycle distribution via CREB through PI3-K dependent pathway in bladder carcinoma cells. Gene, 2012, 496: 8-16

58 Qin R, Chen Z, Ding Y, et al. Long non-coding RNA MEG3 inhibits the proliferation of cervical carcinoma cells through the induction of cell cycle arrest and apoptosis. Neoplasma, 2013, 60: 486-492

59 Alexandrova E M, Moll U M. Role of p53 family members p73 and p63 in human hematological malignancies. Leuk Lymphoma, 2012, 53: 2116-2129

60 Pant V, Quintas-Cardama A, Lozano G. The p53 pathway in hematopoiesis: Lessons from mouse models, implications for humans. Blood, 2012, 120: 5118-5127

61 Zhang X, Zhou Y, Mehta K R, et al. A pituitary-derived MEG3 isoform functions as a growth suppressor in tumor cells. J Clin Endocrinol Metab, 2003, 88: 5119-5126

62 Zhao J, Ohsumi T K, Kung J T, et al. Genome-wide identification of polycomb-associated RNAs by RIP-seq. Mol Cell, 2010, 40: 939-953

63 Zhang X, Gejman R, Mahta A, et al. Maternally expressed gene 3, an imprinted noncoding RNA gene, is associated with meningioma pathogenesis and progression. Cancer Res, 2010, 70: 2350-2358

64 Hoffman B, Amanullah A, Shafarenko M, et al. The proto-oncogene $c-M y c$ in hematopoietic development and leukemogenesis. Oncogene, 2002, 21: 3414-3421

65 Shtivelman E, Bishop J M. Effects of translocations on transcription from PVT. Mol Cell Biol, 1990, 10: 1835-1839

66 Carramusa L, Contino F, Ferro A, et al. The PVT-1 oncogene is a 
Myc protein target that is overexpressed in transformed cells. J Cell Physiol, 2007, 213: 511-518

67 Garding A, Bhattacharya N, Claus R, et al. Epigenetic upregulation of lncRNAs at 13q14.3 in leukemia is linked to the in cis downregulation of a gene cluster that targets NF-kB. PLoS Genet, 2013, 9: e1003373

68 Ratajczak M Z, Shin D M, Schneider G, et al. Parental imprinting regulates insulin-like growth factor signaling: A Rosetta Stone for understanding the biology of pluripotent stem cells, aging and cancerogenesis. Leukemia, 2013, 27: 773-779

69 Argiropoulos B, Palmqvist L, Imren S, et al. Meis1 disrupts the genomic imprint of Dlk1 in a NUP98-HOXD13 leukemia model. Leukemia, 2010, 24: 1788-1791

70 Ripoche M A, Kress C, Poirier F, et al. Deletion of the H19 transcription unit reveals the existence of a putative imprinting control element. Genes Dev, 1997, 11: 1596-1604

71 Gabory A, Ripoche M A, Le Digarcher A, et al. H19 acts as a trans regulator of the imprinted gene network controlling growth in mice. Development, 2009, 136: 3413-3421

72 Iizuka N, Oka M, Tamesa T, et al. Imbalance in expression levels of insulin-like growth factor 2 and $\mathrm{H} 19$ transcripts linked to progression of hepatocellular carcinoma. Anticancer Res, 2004, 24: 4085-4089

73 Esteves L I, Javaroni A C, Nishimoto I N, et al. DNA methylation in the CTCF-binding site I and the expression pattern of the H19 gene: Does positive expression predict poor prognosis in early stage head and neck carcinomas? Mol Carcinog, 2005, 44: 102-110

74 Ariel I, Miao H Q, Ji X R, et al. Imprinted H19 oncofetal RNA is a candidate tumour marker for hepatocellular carcinoma. Mol Pathol, 1998, 51: 21-25

75 Bock O, Schlue J, Kreipe H. Reduced expression of H19 in bone marrow cells from chronic myeloproliferative disorders. Leukemia,
2003, 17: 815-816

76 Tessema M, Langer F, Bock $\mathrm{O}$, et al. Down-regulation of the IGF-2/H19 locus during normal and malignant hematopoiesis is independent of the imprinting pattern. Int J Oncol, 2005, 26: 499-507

77 Takeuchi S, Hofmann W K, Tsukasaki K, et al. Loss of H19 imprinting in adult T-cell leukaemia/lymphoma. Br J Haematol, 2007, 137: 380-381

78 Runge S, Nielsen F C, Nielsen J, et al. H19 RNA binds four molecules of insulin-like growth factor II mRNA-binding protein. J Biol Chem, 2000, 275: 29562-29569

79 Soares M R, Huber J, Rios A F, et al. Investigation of Igf2/Apai and H19/Rsai polymorphisms in patients with cutaneous melanoma. Growth Horm IGF Res, 2010, 20: 295-297

80 Ariel I, Sughayer M, Fellig Y, et al. The imprinted H19 gene is a marker of early recurrence in human bladder carcinoma. Mol Pathol, 2000, 53: 320-323

81 Derrien T, Johnson R, Bussotti G, et al. The GENCODE v7 catalog of human long noncoding RNAs: Analysis of their gene structure, evolution, and expression. Genome Res, 2012, 22: 1775-1789

82 Rinn J L, Chang H Y. Genome regulation by long noncoding RNAs. Annu Rev Biochem, 2012, 81: 145-166

83 Lee C S, Ungewickell A, Bhaduri A, et al. Transcriptome sequencing in Sezary syndrome identifies Sezary cell and mycosis fungoides-associated lncRNAs and novel transcripts. Blood, 2012, 120: 3288-3297

84 Brockdorff N. Noncoding RNA and polycomb recruitment. RNA, 2013, 19: 429-442

85 Landt S G, Marinov G K, Kundaje A, et al. ChIP-seq guidelines and practices of the ENCODE and modENCODE consortia. Genome Res, 2012, 22: 1813-1831

Open Access This article is distributed under the terms of the Creative Commons Attribution License which permits any use, distribution, and reproduction in any medium, provided the original author(s) and source are credited. 